\title{
Antibiotic sensitivity pattern of diarrhoeal pathogens in under five children MK Pervin ${ }^{1}$, ST Jhora ${ }^{2}$, S Paul ${ }^{3}$, A Naher ${ }^{4}$
}

\begin{abstract}
Background: Different bacterial enteropathogens are responsible for diarrhoea in children less than 5 years of age in Bangladesh. This study aimed to determine the antimicrobial sensitivity pattern of diarrhoeal pathogens that is necessary to know for the specific management of acute bacterial diarrhoea.
\end{abstract}

objectives: To find out the bacterial enteropathogens causing diarrhoea and their antimicrobial sensitivity pattern in under 5 children.

\begin{abstract}
Methodology: This was an observational cross sectional study carried out at Microbiology Department of Sir Salimullah Medical College, Dhaka. A total of 217 diarrhoeal patients 0-5 years age, attending Pediatric unit of Sir Salimullah Medical College and Mitford Hospital. Dhaka and Dhaka Shishu Hospital, Dhaka during the period of July, 2015 to June, 2016, were enrolled in the study. For isolation of the common enteric bacteria, diarrhoeal stools were cultured on MacConkey agar media and for Campylobacter spp, diarrhoeal stools were cultured on selective Modified Campy BAP media and nonselective Blood agar media with membrane filter. Positive culture was confirmed by Gram stain morphology and standard biochemical tests. Antimicrobial susceptibility test were used to identify the bacterial sensitivity pattern in the isolates.
\end{abstract}

Results: Out of 217 diarrhoeal stools, bacteria was isolated from 97 (44.70\%) patients by culture. Among 97 culture positive cases Eschericha coli (5152.58\%), Campylobacter spp. (23-23.71\%), Shigella spp. (15-15.46\%) and Salmonella spp. (8-8.25\%) were isolated. Mecillinam is the most sensitive drug for Escherichia coli, Campylobacter spp., Shigella spp. and Salmonella spp. Among the other antimicrobials, Campylabacter spp. were sensitive to azithromycin, erythromycin and nitrofurantoin. Escherichia coli were sensitive to nitrofurantoin, gentamicin and azithromycin. Shigella spp. were sensitive to ceftriaxone, ciprofloxacin and nitrofurantoin and Salmonella spp. were sensitive to ceftriaxone, cotrimoxazole and gentamicin.

Conclusion: Mecillinam and nitrofurantoin were highly effective against bacteria causing diarrhoea in children.

Key words: Diarrhoea, Bacteria, Culture, Sensitivity.

\section{Introduction}

Acute infectious diarrhoea is one of the leading causes of morbidity and mortality among infants and children in developing countries of the world. Diarrhoea remains the second leading cause of death and causes 1.3 million deaths every year in children under 5 years of age. ${ }^{1}$

Diarrhoea defined as excessive and frequent evacuation of watery faeces usually indicating gastrointestinal disease or disorder of 3-7 days duration. ${ }^{2}$ In developing countries nearly 1.4 billion episodes of diarrhoea occur annually in children below 5 years, of which 123.6 million episodes required outpatient medical care and 9 million needed hospitalizations. ${ }^{3}$ In Bangladesh one in fifteen children die before their fifth birth day and among them diarrhoea causes $7 \%$ of death. 4

Aetiology of diarrhoea is variable according to geographic and climate conditions, host factors

1. Mst Khashruba Pervin M Phil, Lecturer, Department of Com. Med, Khulna Medical College (Email: drsurovimicrobiology@gmail.com) 2. Sanya Tahmina Jhora M Phil, Professor and Ex Head, Department of Microbiology, Sir Salimullah Medical College, Dhaka

3. Shikha Paul M Phil, Professor and Head, Department of Microbilogy, Sir Salimullah Medical College, Dhaka

4. Akhtarun Naher M Phil, Professor and Ex Head, Dept. of Microbiology, Sir Salimullah Medical College. Dhaka 
and socioeconomic situations. A wide range of bacteria, virus and parasites causes infectious diarrhoea. 5 Among the bacterial causes, Escherichia coli is most common pathogen for childhood diarrhoea in developing countries and now being an emerging antimicrobial resistant enteropathogen in developed countries. ${ }^{6}$ In Bangladesh, about $41-46 \%$ acute diarrhoea is caused by Escherichia coli7 Worldwide Campylobacter spp. is one of a major cause of acute bacterial gastroenteritis among infants and children. 8 In Bangladesh isolation rate of Campylobacter within 5 years of age is $11 \%$ to $17.4 \% .{ }^{9}$ Shigellosis is endemic in Bangladesh and it is estimated that $2,00,000$ to $3,00,000$ cases of shigellosis are found per year.10 For earlier diagnosis and specific treatment for diarrhoea, it is very important to know the exact pathogen and its antimicrobial sensitivity pattern. But currently antibiotic resistance has been increasing and is a major threat to public health. The study was designed to find out bacteria and its antimicrobial sensitivity pattern from children suffering from diarrhoea under 5 years of age.

\section{Material and methods}

This was an observational cross sectional study. The study protocol was approved by Ethical Review Committee of Sir Salimullah Medical College and Mitford Hospital, Dhaka. The study was conducted from July, 2015 - June, 2016 in Microbiological laboratory of Sir Salimullah Medical College, Dhaka.

During this period a total of 217 stool samples were collected from acute diarrhoeal children upto 5 years of age in Pediatric unit of Sir Salimullah Medical College and Mitford Hospital and Dhaka Shishu Hospital, Dhaka. Stools were collected in a clean dry. wide mouth and leak proof container and brought to the Microbiological laboratory, of Sir Salimullah Medical College, Dhaka within 2 hours. The selected cases were the children upto 5 years of age passing watery or loose stools three or more times in 24 hours with/without mucus and or blood, vomiting, fever abdominal pain. Children on antibiotic or completed antibiotic treatment within last 15 days were excluded.

Stools were examined microscopically in wet film preparation for detection of pus cells. Then all samples were plated on MacConkey agar media and incubated at $37^{\circ} \mathrm{C}$ for 24 hours in aerobic condition for isolation of enteric bacteria. For isolation of Compylobacter spp. stool samples were inoculated in Selective Modified Campy BAP media (Brucella agar base with 10\% defibrinated sheep blood and 3 antimicrobials Polymixin B 2.5 $\mathrm{IU} / \mathrm{ml}$, Amphotericin B 2.0mg/liter and Vancomycin $10 \mathrm{mg} /$ liter) and Non selective Blood agar media with membrane filter (0.45 um). A cellulose triacetate membrane with 0.45 um pore and $47 \mathrm{~nm}$ diameter were placed on surface of Blood ager plate before inoculation. After inoculation media were incubated in candle jar at $42^{\circ} \mathrm{C}$ for 48 hours. Suspected colonies were identified by colony character, Gram stain and standard biochemical methods. Susceptibility to antimicrobial agents was done by Kirby Bauer modified disk diffusion technique using Mueller. Hinton agar with $5 \%$ sheep blood was used for Campylobacter spp. Mueller Hinton agar was used for other enteric bacteria.

The data of the patients were collected on a structured questionnaire. All data were compiled and analyzed using Microsoft excels (2007) and presented in table to describe it in percentage. As the values were small, comparison between them could not be done.

\section{Results}

During the study period from July, 2015 to June, 2016 a total of 217 stool samples were collected from under 5 years children with diarrhoeal disease.

Table-1 shows isolation of bacteria from diarrhoeal stools. Of the 217 samples, bacteria were isolated in $97(44.70 \%)$ samples. Among the culture positive 97 samples, Escherichia coli were isolated in $51(52.58 \%)$ sample, Campylobacter spp. were isolated in $23(23.71 \%)$ samples, Shigella spp. were isolated in $15(15.46 \%)$ samples and Salmonela spp. were isolated in $8(8.25 \%)$ samples. No bacteria were isolated in $120(55.30 \%)$ samples.

\section{Table I}

Isolation of bacteria from diarrheal stools by culture $(n=217)$

\begin{tabular}{lc}
\hline Name of isolated bacteria & No. of cases $(\%)$ \\
\hline Culture positive cases & $97(44.70)$ \\
Escherichia coli & $51(52.58)$ \\
Campylobacter spp. & $23(23.71)$ \\
Shigella spp. & $15(15.46)$ \\
Salmonella spp. & $08(08.25)$ \\
Culture negative cases & $120(55.30)$ \\
\hline Total & $217(100)$ \\
\hline
\end{tabular}

Table II shows sensitivity pattern Escherichia coli to different antimicrobials by disc diffusion method. Among these tested antimicrobials, E 
coli were highly susceptible $(100 \%)$ to mecillinam followed by nitrofurantoin $(84.32 \%)$, gentamicin $(88.24 \%)$ and cotrimoxazole $(76.47 \%)$. Moderately sensitive to azithromycin (50.98\%), less sensitive to ceftriaxone $(41.18 \%)$ and ciprofloxacin (39.22\%). E coli were highly resistant to ampicillin (96 08\%), erythromycin (90.2\%) and nalidixic acid (80.39\%).

\section{Table II}

Sensitivity pattern of Escherichia coli to different antimicrobials $(n=51)$

Name of antimicrobials No. of isolates (\%) Sensitive(S) Intermediate(I) Resistant(R)

\begin{tabular}{lccc}
\hline Ampicillin & $2(3.92)$ & 00 & $49(96.08)$ \\
Erythromycin & $5(9.80)$ & 00 & $46(90.20)$ \\
Nalidixic acid & $7(13.73)$ & $3(5.88)$ & $41(80.39)$ \\
Cotrimoxaole & $18(35.29)$ & 00 & $33(64.71)$ \\
Ciprofloxacin & $20(39.22)$ & $9(17.64)$ & $22(43.14)$ \\
Azithromycin & $26(50.98)$ & 00 & $25(49.02)$ \\
Ceftriaxone & $21(41.18)$ & $2(3.92)$ & $28(54.90)$ \\
Gentamicin & $41(80.40)$ & $5(9.80)$ & $5(9.80)$ \\
Nitrofurantoin & $43(84.32)$ & $4(7.84)$ & $4(7.84)$ \\
Mecillinam & $51(100)$ & 00 & 00 \\
\hline
\end{tabular}

Table-III shows sensitivity pattern of Campylobacter spp. to different antimicrobials. In this study Campylobacter spp. were highly sensitive $(100 \%)$ to gentamycin and mecillinam followed by azithromycin (95.65\%) erythromycin (86.96\%) and nitrofurantoin (78-26\%). Moderately sensitive to nalidixic acid (62.5\%) and ciprofloxacin $(73.91 \%)$. campylobacter spp. were highly resistant to cotrimoxazaole (91.3\%) followed by ampicillin (82.61\%) and ceftriaxone (65.22\%).

\section{Table III}

Sensitivity pattern of Campylobacter spp. to different antimicrobials $(n=23)$

No. of isolates (\%)

Name of antimicrobials

Sensitive(S) Intermediate(I) Resistant(R)

\begin{tabular}{lccc}
\hline Ampicillin & $4(17.39)$ & 00 & $19(82.61)$ \\
Erythromycin & $20(86.96)$ & 00 & $3(13.04)$ \\
Nalidixic acid & $15(65.23)$ & 00 & $8(34.78)$ \\
Cotrimoxazo1e & $2(8.70)$ & 00 & $21(91.30)$ \\
Ciprofloxacin & $17(73.91)$ & 00 & $6(26.09)$ \\
Azithromycin & $22(95.65)$ & 00 & $1(4.35)$ \\
Ceftriaxone & $7(30.43)$ & $1(4.35)$ & $15(65.22)$ \\
Gentamicin & $23(100)$ & 00 & 00 \\
Nitrofurantoin & $18(78.26)$ & 00 & $5(21.74)$ \\
Mecillinam & $23(100)$ & 00 & 00 \\
\hline
\end{tabular}

Table IV shows sensitivity pattern of Shigella spp. to different antimicrobials by disc diffusion method. In this study, Shigella spp. were highly sensitive $(100 \%)$ to mecillinam followed by ceftriaxone (86.67\%) and ciprofloxacin (80\%). Moderate to low rate of sensitivity to nitrofurantoin (73.34\%), azithromycin (73.34\%) and gentamicin (53.34\%); Highly resistant to erythromycin (93.34\%), cotrimoxazole (86.67\%), ampicillin (80\%) and nalidixic acid (60\%).

\section{Table IV}

Sensitivity pattern of Shigella spp. to different antimicrobials $(n=15)$

\begin{tabular}{lccc}
\hline \multicolumn{4}{c}{ No. of isolates(\%) } \\
Name of antimicrobials \\
\multicolumn{4}{c}{ Sensitive (S) Intermediate (I) } \\
\hline Ampicistant(R) \\
Erythromycin & $3(20)$ & 00 & $12(80)$ \\
Nalidixic acid & $1(6.66)$ & 00 & $14(93.34)$ \\
Cotrimoxazole & $2(13.33)$ & 00 & $13(86.67)$ \\
Ciprofloxacin & $12(80)$ & $2(13.33)$ & $1(6.67)$ \\
Azithromycin & $11(73.34)$ & 00 & $4(26.66)$ \\
Ceftriaxone & $13(86.67)$ & 00 & $2(13.33)$ \\
Gentamicin & $8(53.34)$ & $2(13.33)$ & $4(33.33)$ \\
Nitrofurantoin & $11(73.34)$ & $1(6.66)$ & $3(20)$ \\
Mecillinam & $15(100)$ & 00 & 00 \\
\hline
\end{tabular}

Table V shows sensitivity pattern of Salmonella spp. to different antimicrobials by disc diffusion method. In this study, Salmonella spp. were

\section{Table V}

Sensitivity pattern of Salmonella spp. to different antimicrobials $(\mathrm{n}=8)$

No. of isolates (\%)

Name of antimicrobials

Sensitive(S) Intermediate(I) Resistant(R)

\begin{tabular}{lccc}
\hline Ampicillin & $5(62.5)$ & 00 & $3(37.5)$ \\
Erythromycin & 00 & 00 & $8(100)$ \\
Nalidixic acid & 00 & 00 & $8(100)$ \\
Cotrimoxazole & $7(87.5)$ & 00 & $1(12.5)$ \\
Ciprialoxacin & $4(50)$ & $2(25)$ & $2(25)$ \\
Azithromycin & $2(25)$ & 00 & $6(75)$ \\
Ceftrkxone & $8(100)$ & 00 & 00 \\
Gentamicin & $7(78.5)$ & 00 & $1(12.5)$ \\
Nitroflrantoin & $6(75)$ & $1(12.5)$ & $1(12.5)$ \\
Mecillinam & $8(100)$ & 00 & 00 \\
\hline
\end{tabular}


highly sensitive (100\%) to ceftriaxone and mecillinam, followed by cotrimoxazole $(87.5 \%)$ and gentamicin (87.5\%). Among the other antimicrobials sensitivity pattern were as follows: nitrofurantoin (75\%), ampicillin (62.5\%), ciprofloxacin (50\%) and azithromycin (25\%). Salmonella spp. were $100 \%$ resistant to erythromycin and nalidixic acid.

\section{Discussion}

Diarrhoeal diseases still remains as a second leading cause of child death causing 1 in every 5 death in developing world. In Southeast Asia, child mortality rate is high $(31.3 \%)$ due to diarrhoea. ${ }^{11}$ In this study, out of 217 stool samples, enteric bacteria were isolated in $44.7 \%$ samples. Among these diarrhoeal pathogens Escherichia coli was the most predominant $(52.58 \%)$ bacteria in this study. Like Bangladesh Escherichia coli is the most common aetiology of diarrhoea in other developing countries like India (44.2\%) and Sudan (53.8\%). ${ }^{12,13}$

Campylobacter spp. were isolated as second prevalent diarrhoeal pathogen (10.6\%) from diarrhoeal stool in this study. In the present study, Shigella spp. was isolated $(15.46 \%)$ from diarrhoeal stool. In Bangladesh incidence of shigellosis is higher in winter (October-January) and $(9.20 \%)$ Shigella was isolated in another study.6 Frequency of isolation of Shigella spp. $(36.8 \%)$ were higher in other developing countries like Nepal.14 In present study the percentage of Salmonella spp. was $8.25 \%$. Nessa reported $16 \%$ Salmonella spp. which was higher than present study. 15

Current study showed that E. coli were susceptible to mecillinam, nitrofurantoin and gentamicin above $80 \%$. Resistance of ampicillin and cotrimoxazole were reported in previous study which agreed with the present study. ${ }^{16}$ Sensitivity to azithromycin, ciprofloxacin and ceftriaxone were low. The high incidence of antibiotic resistance isolates of $\mathrm{E}$. coli may be due to indiscriminate use of antibiotics. ${ }^{17}$

Most of the Campylobacter infections do not require antimicrobial treatment since they are clinically mild and self limiting in nature. Fewer than $50 \%$ need antimicrobial treatment. In the present study, Campylobacter was $100 \%$ sensitive to gentamicin, $95.65 \%$ to azithromycin. $86.96 \%$ to erythromycin, $73.91 \%$ to ciprofloxacin. Marked resistance to ciprofloxacin in both developed and developing countries as a result of inappropriate and indiscriminate, use of quinolones. Macrolides are the drug of choice for Campylobacter enteritis and intravenous aminoglycosides for systemic manifestations. ${ }^{18}$ So, a specific sensitivity pattern guideline is needed for the treatment of enteric and systemic campylobacteriosis.

Among 15 Shigella spp. all isolates were sensitive to mecillinam followed by ceftriaxone, ciprofloxacin and azithromycin that showed similarity with Rahman. ${ }^{19}$ An increasing resistance had been reported in developing countries including Bangladesh to ampicillin, cotrimoxazole and nalidixic acid. ${ }^{20}$

Out of 8 Salmonella spp. all (100\%) were sensitive to ceftriaxone and mecillinam. Nessa reported that Salmonella spp. were highly sensitive to ciprofloxacin (100\%) and less in cotrimoxazole $(40 \%)$ but that did not match with the present study. ${ }^{21}$ In the present study, ciprofloxacin were $50 \%$ and cotrimoxazole were $87.5 \%$ sensitive to Salmonella spp. $75 \%-100 \%$ isolates were resistant to azithromycin, erythromycin and nalidixic acid. So, the overall sensitivity pattern of antibiotics varies from country to country according to prescription of local physician and also on quality of discs and methods used.

\section{Conclusion}

Mecillinam and nitrofurantoin were highly effective antibiotic against diarrhoea in children. As the antibiotic sensitivity pattern to bacterial pathogens is changing, so stool culture should be done before treatment. Sensitivity pattern of diarrhoeal pathogens specify treatment and shortens sufferings of child.

\section{References}

1. Black RE, Cousens S, Johnson HL. For the Child Health Epidemiology Reference Group of WHO and UNICEF. Global, regional and national cause of child mortality in 2008: a systematic analysis. Lancet 2010; 375: 1969-87

2. Thapar N, Sanderson IR. Diarrhoea in children : an interferance between developing and developed countries Lancet 2004; 363: 641-53

3. Kosek M, Bern C, Guerrant R. L The Global Burden of Diarrhoeal Disease, as Estimated from Studies Published between 1992 and 2000. Bulletin of the World Health Organization. 2003; 81: 197-204

4. Bangladesh demographic and health survey (BDHS), 2007; Available at: http//w.w.w.measuredhs. cotn/pubs

5. WGO. World Gastroenterology Organization Practice Guideline: Acute diarrhea Available at http: /www.omge.org/global guideline,htm.2008. 
6. Akinjogunl OJ, Eghafona NO, Ekoi OH. Diarrlioeagenic Escherichia Coli (DEC): prevalence among in and ambulatory patients and susceptibility to antimicrobial chemotherapeutic agents. J Bacterial Res 2009; 1: 34-38

7. Nessa K, Ahmed D, Islam J, Kabir FML and Hossain MA. Usefulness of a Multiplex PCR for Detection of diarrhoeagenic Escherichia coli in a diagnostic microbiology laboratory setting Bangladesh. J.Med Microbiol 2007; 1: 38-42

8. Friedman CR, Hoekstra RM, Samuel M. Marcus R. Bender $J$ et al. Risk Factors for Sporadic Campylobacter Infection in the United States: A Case Control Study in FoodNet Sites. Clin Infection; Dis 2004; 38: 285-96

9. Talukder KA, Aslam M, Islam Z. Azmi IJ, Datta DK, Hossain $S$ et al. Prevalence of Virulence Genes and Cytolethal Distending Toxin Production in Campylobacter jejuni Isolates from Diarrheal Patients in Bangladesh. Journal of Clinical Microbiology 2008: 46: 1485-88

10. National Encyclopedia of Bangladesh 2010. Available at htm://www. banglapedia.org/httdocs/HT/-0336.HTM accessed on 4.11 .2015

11. Walker CL, Aryee MJ, Boschi Pinto C., Black RE Estimating diarrhea mortality among young children in low and middle income countries. PLos One 2012; 7: C29151

12. Rauther VK, Pathana M. Jayara A. Yadav N. Clinical study of acute childhood diarrhea by bacterial enteropathogens. J Clin Diagn Res. 2014: 8: 1-5.

13. Saseed A, Abd $h$ and Sandstrom G. Micribial aetiology of acute diarrhea under five years of age in Khartoum Sudan. J Med Microbiology. 2015; 64: 432-437
14. Lama C and Sherchan JB. Enteropathogens associated diarrhea in hospitalized patients of Children's Hospital, Kathmandu, J Nepal Heal Res Coun 2006; 5: 50-57

15. Nessa MK, Khan MSR and Alam. Isolation, identification and characterization of Salmonella serovars from diarrhoeic stool samples of human. Bangl. J. Vet. Med. 2011; 9: 85-93.

16. Aslani MM, Ahrabi SS, Alikhani YM. Molecular detection and antimicrobial resistance of diarrheagenic, Escherichia coli strains isolated from diarrhoeal cases, Saudi Med J 2008; 29: 388-392

17. Chakraborty S, Deokule JS, Garg P, Bhattacharya SK, Nandy RK, Nair GB et al. Concimitent infection of enterotoxigenic E. coli in an outbreak of cholera caused by Vibrio cholerae 01 and 0139 in Ahmedabad, India. J Clin Microbiol 2001; 39: 3241 49

18. Gaudreau, C., and H. Gilbert. Antimicrobial resistance of clinical strains of Campylobacter jejuni subsp. jejuni isolated from human in 1998 to 2001 in Montreal, Canada. Antimicrob. Agents and Chemotherapy 2003, 2027-29

19. Rahman M, Shoma S, Rashid H, Arifeen S et al. Increasing spectrum in antimicrobial resistance of Shigella isolates in Bangladesh: Resistance to aziythromycin and ceftriaxone and decreased susceptibility to ciprofloxacin. J health popul nutr. 2007; 25: 158-67

20. Fulla N, Prado V, Duran C. Lagos R, Levine MM. Surveillance for antimicrobial resistance profiles among Shigella species isolated from a semirural community in the northern administrative area of Santiago, Chile Am J Trop Med. 2005; 72: 851-54

21. Nessa MK, Khan MSR and Alam Isolation, identification and characterization of Salmonella serovars from diarrhoeic stool samples of human. Bangl J Vet Med 2011: 9: 85-93 